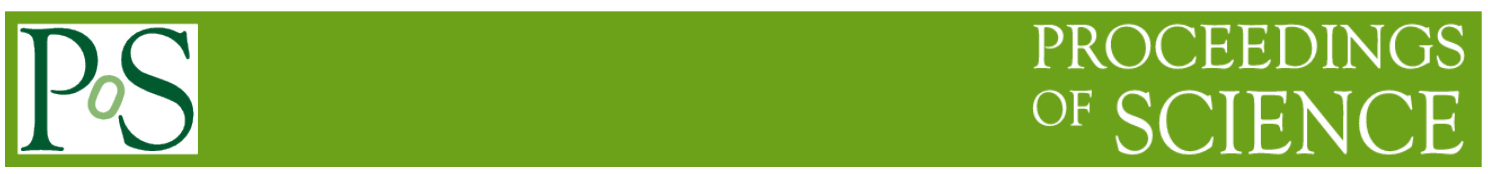

\title{
Detection of Low-Weight Pigs by Using a Top-View Camera
}

\section{Jaewon Sa ${ }^{1}$}

Dept. of Computer and Information Science, Korea University, Sejong, 339-700, Korea

E-mail: sjwon92@korea.ac.kr

\section{Miso Ju}

Dept. of Computer and Information Science, Korea University, Sejong, 339-700, Korea

E-mail: misoalth@korea.ac.kr

\section{Seoungyup Han}

Dept. of Computer and Information Science, Korea University, Sejong, 339-700, Korea

E-mail: hansy910korea.ac.kr

\section{Heegon Kim}

Dept. of Computer and Information Science, Korea University, Sejong, 339-700, Korea

E-mail: khg860korea.ac.kr

\section{Yongwha Chung ${ }^{23}$}

Dept. of Computer and Information Science, Korea University, Sejong, 339-700, Korea

E-mail: ychungyekorea.ac.kr

\section{Daihee Park}

Dept. of Computer and Information Science, Korea University, Sejong, 339-700, Korea

E-mail: dhparkekorea.ac.kr

Caring weaning pigs is important in the management of a group-housed pig farm. In this study, we propose an automatic method for detecting low-weight pigs in a pigsty. We install a top-view camera in a room of weaning pigs to detect the motion area of each pig from the video obtained. Then, we automatically detect a low-weight pig by comparing the size of each pig. Based on the experimental results, we confirm that the proposed method can automatically detect relatively low-weight pigs without any manual inspection or measurement of actual weight by a farm administrator.

ISCC 2015

18-19, December, 2015

Guangzhou, China

\footnotetext{
${ }^{1}$ Speaker

${ }^{2}$ Corresponding Author

${ }^{3}$ This research was supported by Basic Science Research Program through the NRF funded by the MEST (2015R1D1A1A09060594) and BK21 Plus Program.
} 


\section{Introduction}

Caring weaning animals is important in the management of group-housed livestock[1-9]. For example, if one of the weaning pigs has a lower weight when compared to other pigs, it may have some health problem caused by a disease or stress; therefore, a farm administrator should rapidly identify the low-weight pig and take necessary steps to the pig. In case of a large-scale Korean pigsty, however, each farm administrator has to manage 2,000 pigs. Under this condition, it is impossible for the administrator to detect low-weight pigs early; in this sense, the automatic detection of low-weight pigs is required.

In this study, we propose an automatic method for detecting low-weight pigs in a pigsty. We install a top-view camera in the ceiling of a pig room to automatically detect low-weight pigs by measuring the size of each pig with computer vision techniques. That is, we detect the moving area of pigs obtained from the video and compare the relative size of each pig. In fact, the weaning pigs sleep most of the time during a day, and it is difficult to measure the size of each pig due to occlusion between them. The motivation of this paper is to determine the size of a moving pig by using a motion detection method.

From the input video, we first detect object areas by using HSV color information. If the size of a detected area is larger than that of one pig, we consider that area, as a group of touching pigs which are close to each other. In order to determine the size of each pig in a crowded pig room, we apply Gaussian Mixture Model (GMM) for detecting the moving pigs. Since a pig can move part of his body (i.e., head shaking), GMM may detect partial-moving pigs in addition to full-moving pigs. If the size of an overlapped area between a GMM moving area and the corresponding HSV object area is larger than the threshold as determined experimentally, we consider it as a full-moving pig and record its size. Finally, if the size of a full-moving pig is less than a threshold (i.e., the size of a pig is significanlty smaller than that of other pigs), we report the pig as a low-weight pig. Thus, our proposed method can detect lowweight pigs automatically without any user's intervention (i.e., visual inspection, measurement of actual weight and camera measurement in a specially organized measurement room). Based on the experimental results, we confirm that the proposed method can automatically detect relatively low-weight pigs.

The remainder of this paper is organized as follows. In Section 2, we present related works with computer vision techniques. In Section 3, we explain the proposed method for measuring the size of a full-moving pig, and we present our experimental results in Section 4. In Section 5, we provide a summary of this study.

\section{Related Works}

Various methods have been reported for managing pigs effectively in a pigsty with computer vision techniques. For example, a study for providing the best temperature to the pigs was proposed by deciding the density of sleeping pigs in a pigsty with a camera installed at the ceiling[2]. For analysis of pig behaviors by using the video informations, a study available of detecting an emergency or a disease occurence early of pigs was proposed[3]. A study which was automatically detected for agressive activities of pigs by using video informations and SVM was reported[4], and a tracking method by using the "frame difference" was reported for detecting and tracking the pigs individually[5]. In the tracking technique by using the frame 
difference, however, pigs could be not tracked accurately because of no segmentation. To solve the problem, a segmentation method was proposed for separating the touching pigs by using spatiotemporal informations[6]. Additionally, researches that estimate the group-housed livestock have been reported. For exmaple, a research that investigated the possibility of estimating the body size and weight of sheep with computer-assisted digital image analysis was conducted[7]. For weighing pigs, a research for pig weighing based on the infrared depth map images was performed[8]. In addition, a research was conducted based on computer-assisted visual images and artificial neural networks for pig live weights without disturbing the animals[9]. In these researches, however, the proposed methods measured the weight of only one of the livestocks in a specially organized measurement room (i.e., requiring some user intervention). In this paper, we propose an automated method for detecting low-weight pigs by using a video information obtained from a top-view camera in a crowded pig room, without any user's intervention.

\section{Detection of Low-weight Pigs}

In this paper, we propose an automated method for detecting low-weight pigs by measuring sizes of moving pigs. First, we perform binarization of a frame for conducting background subtraction from a video obtained by a camera. In the binary image, we also detect the moving pigs by using GMM. If the moving pigs are detected in the video sequence, we calculate an average size of the moving pigs after analyzing the number of pixels of each moving pig (i.e., size of each pig). Finally, we determine the low-weight pigs based on a threshold using the average. Fig. 1 shows the flowchart of the proposed method.

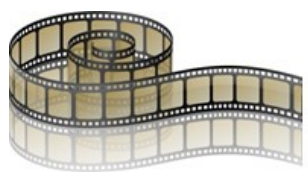

Video Stream

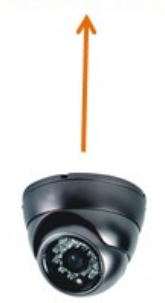

Video Sensor

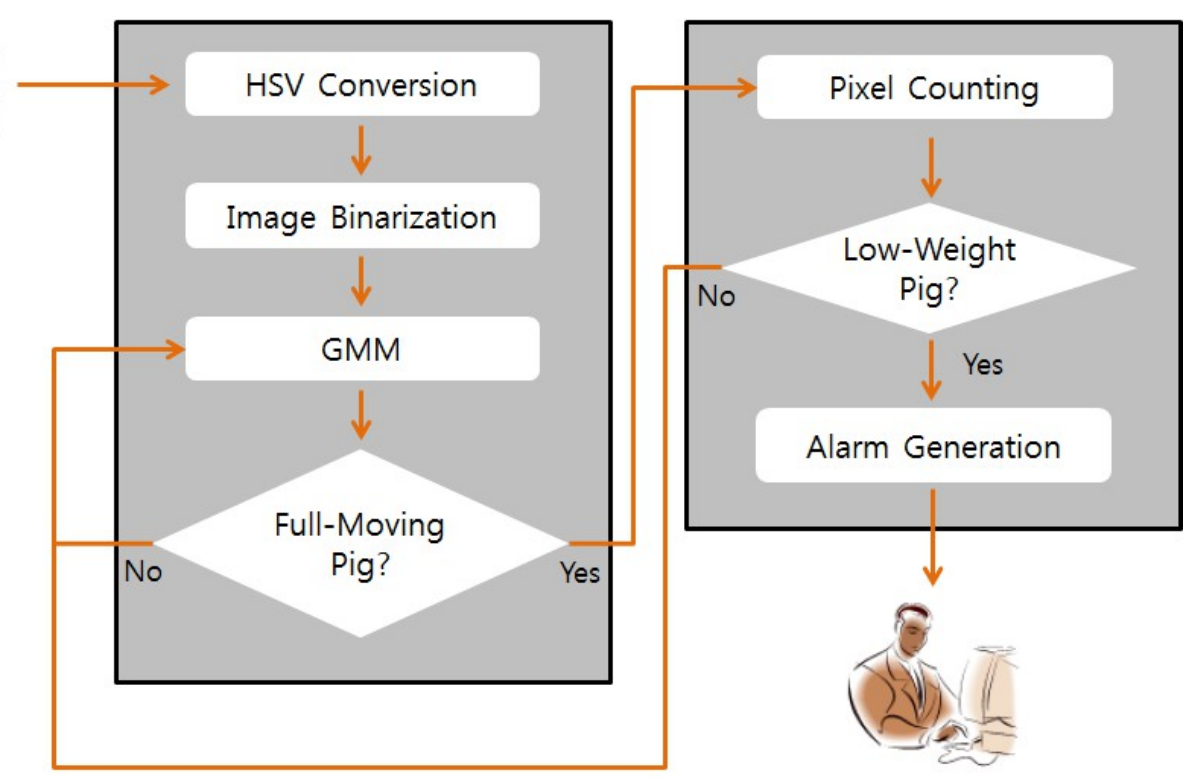

Pigsty Administrator

Figure 1: Flowchart of Detecting Low-weight Pigs 


\subsection{Background Subtraction}

As shadows of the pigs are bulit to similar size near the pigs due to the influence of light in a pig room, a pig can be recognized as two pigs in pre-processing for the RGB input video. To solve this problem, we convert RGB color space to HSV color space to reduce the influence of the light. In addition, we tune parameters of HSV, and perform binarization with the HSV image to remove unnecessary noise. From the binarization, we exclude object areas larger than the size of one pig (i.e., single-pig-size-threshold). For the purpose of explaination, we denote this result as HSV threshold image.

\subsection{Detecting a Moving Pig by Using GMM and Calculating its Size}

GMM method is used to detect moving pigs in the HSV threshold image. Typically, the frame difference is widely used to detect moving objects. This is a simple but efficient background subtraction algorithm to identify the difference of each pixel between the previous frame and the current frame. To detect moving pigs in our experiment, open source GMM[10] is employed. GMM is used for background modeling to subtract the background using each pixel modeled as mixed Gaussiand distributions. In GMM method, it has to learn of the video sequence to detect moving pigs. After learning of the sequence, only moving pigs appear except sleeping or non-moving pigs in the result sequence; however, if one of the pigs in the sequence moves its head or part of its body, it also appears in the result sequence. In the case, we cannot measure the precise size of a pig because of partial motion of a pig. Thus, a full-moving pig should be detected to measure the precise size of it by excluding the partial-moving pigs. For detecting the full-moving pig, overlapping the HSV threshold image and the GMM image is required. A full-moving pig is determined by counting the number of pixels in the the HSV threshold image overlapped with the GMM image. In order to detect a full-moving pig, a threshold can be set from the maximum size among each moving pig. If the number of overlapped pixels is larger than the threshold (i.e., full-moving-pig-threshold) of the number of pixels in the HSV threshold image, the pig can be detected as a full-moving pig. Then, we analyze the number of pixels of each full-moving pig after deriving an average size of fullmoving pigs. At the time, a threshold for detecting a low-weight pig can be derived statistically from the average size of the full-moving pigs. Based on this procedure, if the size of a fullmoving pig is larger than the threshold (i.e., low-weight-pig-threshold), the pig is determined as a low-weight pig.

\section{Experimental Results}

In our experiments, we set the resolution size to $640 \times 480$ pixels and the frame rate to 8 frames/second. The camera was located at $4 \mathrm{~m}$ above the floor to monitor a pig room of $4 \times 3 \mathrm{~m}$, and there were 22 weaning pigs in the room. Firstly, we performed the background subtraction to remove some noise of the input video sequence. Fig. 2 shows the result image of converting a RGB color image to a HSV binary image from the video. 


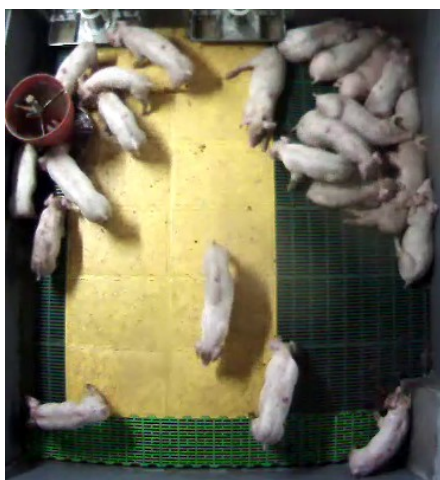

(a) RGB Color Image

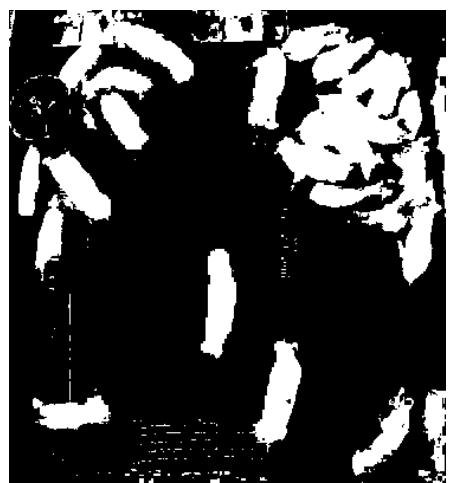

(b) Result of Binarization

Figure 2: Binarization of Input Image

From the binarization, we excluded object areas larger than the size of one pig (i.e., singlepig-size-threshold). In the experiments, we set the single-pig-size-threshold as $110 \%$ of the maximum size of the pig, as shown in Fig. 3 (a). Then, we used the GMM method to detect the moving pigs in the HSV threshold image. When a pig moved a part of its body, however, partial-moving pigs appeared as well as full-moving pigs as shown in Fig. 3 (b). We overlapped the GMM image with the HSV threshold image, and detected full-moving pigs as shown in Fig. 4. In the experiments, we set the full-moving-pig-threshold as $90 \%$ of the size of the corresponding pig in the HSV threshold image.

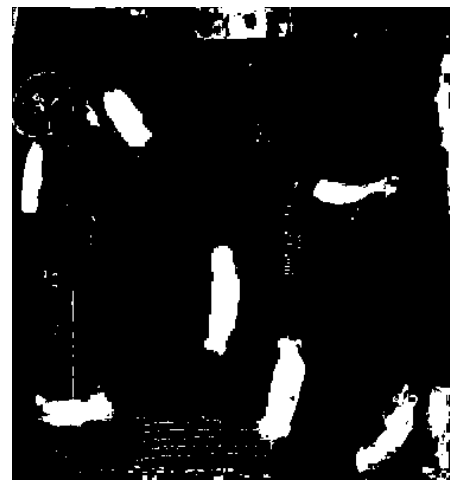

(a) HSV Threshold Image

Figure 3: Detection of Moving Pigs

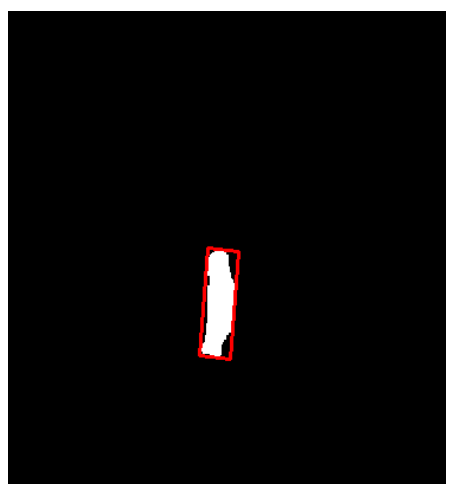

(a) HSV Threshold Image

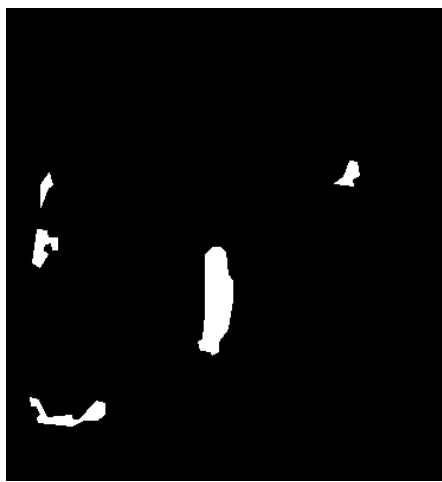

(b) Result Image of GMM

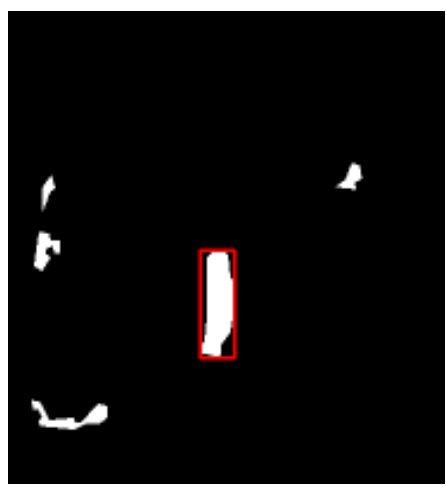

(b) Result of GMM

Figure 4: Detection of A Full-moving Pig 
Finally, we detected the low-weight pigs. From the 6-minute input video, we detected 10 full-moving pigs. Then, we counted the number of pixels of each full-moving pig. To find out low-weight pigs, we derived an average by summing the size (i.e., the number of pixels) of each full-moving pig in the video sequence. We set the low-weight-pig-threshold as $70 \%$ of the average. In our experimental results with 10 full-moving pigs, we identified that pig \#10 was especially smaller than any other pig. Fig. 5 shows the low-weight-pig-threshold, and pig \#10 was detected as a low-weight pig. According to the experimental results, the advantage of our proposed method was measurable for the size of the moving pigs in the crowded pig room compared to measure the size of a pig in a specially organized measurement room.

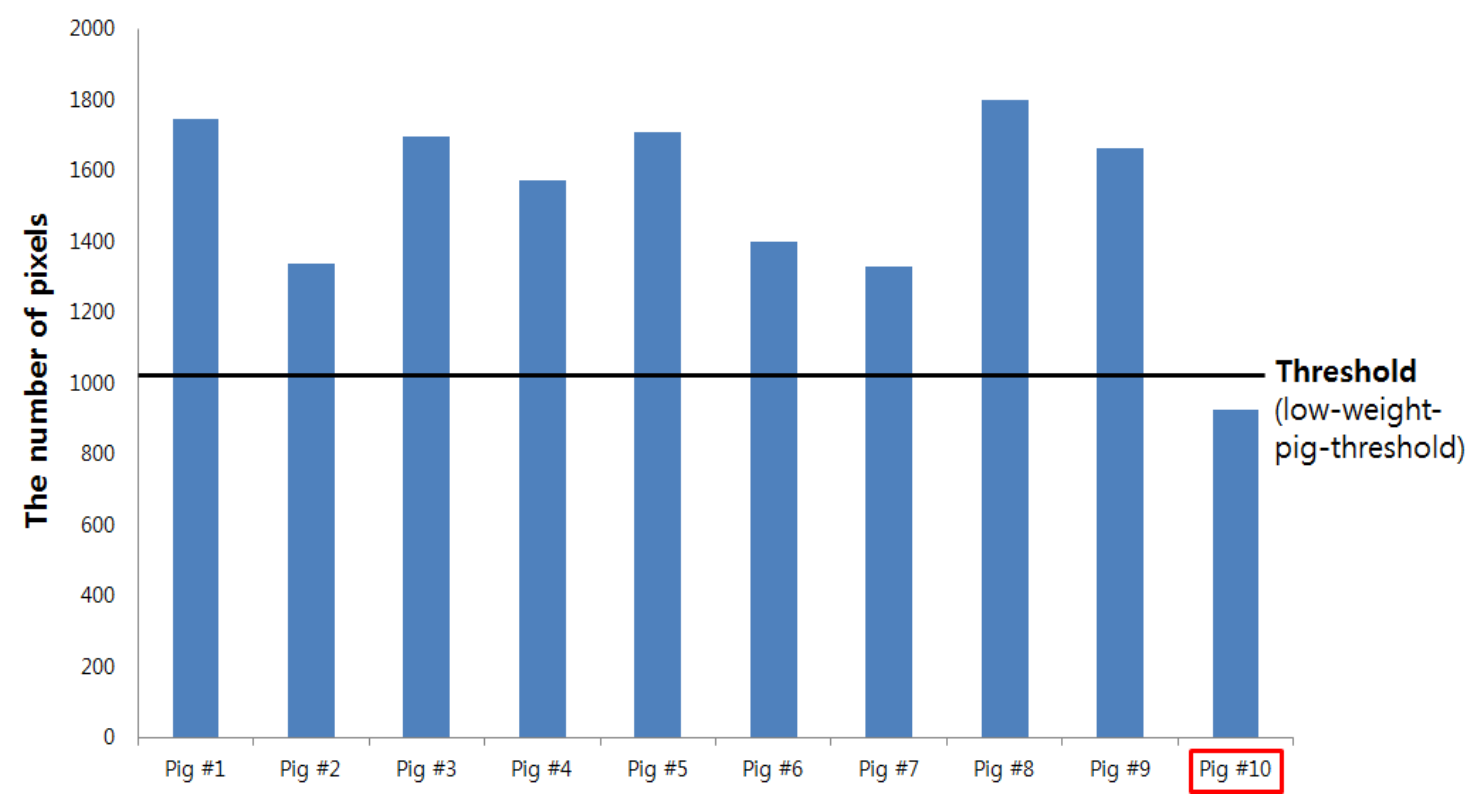

Figure 5: number of Pixels of 10 Pigs in the Video Sequence

\section{Conclusion}

Detection of low-weight weaning pigs is important in livestock management because it allows early detection of the health or management problems. In this paper, we proposed an automated method for detecting low-weight pigs, which could significantly save the working time of farm administrators. From the top-view camera installed at the ceiling of a pig room, we analyzed the size of each pig with computer vision techniques to detect relatively low-weight pigs in the pig room. From the experimental results, we confirm that the proposed method can automatically detect low-weight pigs by measuring the moving area of each pig without any user's intervention. We expect that weaning pigs can be more effectively managed if the proposed method is combined with future work of 24-hour tracking of each pig.

\section{References}

[1] T. Banhazi, H. Lehr, J. Black, H. Crabtree, P. Schofield, M. Tscharke, and D. Berckmans. Precision Livestock Farming: an International Review of Scientific and Commercial Aspects[J]. International Journal of Agriculture and Biology. 5(1): 1-9(2012)

[2] A. Nasirahmadi U. Richter, O. Hensel, S. Edwards and B. Sturm. Using Machine Vision for Investigation of Changes in Pig Group Lying Patterns[J]. Computer and Electronics in Agriculture. 119(1): 184-190(2015) 
[3] Y. Chung, H. Kim, D. Park, T. Jeon, and H. H. Chang. A Cost-Effective Pigsty Monitoring System based on a Video Sensor[J]. KSII Tr. Internet \& Info. Systems. 8(4): 1481-1498(2014)

[4] L. Jin, S. Zuo, J. Lee, D. Park, and Y. Chung. Aggressive Behavior Detection of Weaning Pigs[C]. Proc. of KSII Fall Conference. KSII, Seoul. 15(2): 325-326(2014)

[5] S. Zuo, L. Jin, Y. Chung, and D. Park. An Index Algorithm for Tracking Pigs in Pigsty[C]. Proc. of ICITMS2014. WIT Transaction on Engineering Sciences, Southampton. 93(1): 797-803(2014)

[6] S. Han, S. Lee, J. Sa, H. Kim, S. Lee, Y. Chung, and D. Park. Segmentation of Touching Pigs using Spatiotemporal Information[C]. Proc. of KIPS Spring Conference. KIPS, Seoul. 1(1): 866$869(2015)$

[7] P. Menesatti, C. Costa, F. Antonucci, R. Steri, F Pallottino, and G. Catillo. A Low-cost Stereovision System to Estimate Size and Weight of Live Sheep [J]. Computers and Electronics in Agriculture. 103(1): 33-38(2014)

[8] J. Kongsro. Estimation of Pig Weight using a Microsoft Kinect Prototype Imaging System[J]. Computers and Electronics in Agriculture. 109(1): 32-35(2014)

[9] A. Wongsriworaphon, B. Arnonkijpanich, S. Pathumnakul. An Approach based on Digital Image Analysis to Estimate the Live Weights of Pigs in Farm Environments[J]. Computers and Electronics in Agriculture. 115(1): 26-33(2015)

[10] Open Source Computer Vision. OpenCV. http://opencv.org 